\title{
Function and dysfunction of leucine-rich repeat kinase 2 (LRRK2): Parkinson's disease and beyond
}

\author{
Jae Ryul Bae ${ }^{1} \mathcal{E}$ Byoung Dae Lee $e^{1,2,3, *}$ \\ ${ }^{1}$ Department of Neuroscience, ${ }^{2}$ Neurodegeneration Control Research Center, ${ }^{3}$ Department of Physiology, School of Medicine, Kyung Hee \\ University, Seoul 130-701, Korea
}

\begin{abstract}
Mutations in leucine-rich repeat kinase 2 (LRRK2) are the most common cause of familial Parkinson's disease (PD). As such, functions and dysfunctions of LRRK2 in PD have been the subject of extensive investigation. In addition to PD, increasing evidence is suggesting that LRRK2 is associated with a wide range of diseases. Genome-wide association studies have implicated LRRK2 in Crohn's disease (CD) and leprosy, and the carriers with pathogenic mutations of LRRK2 show increased risk to develop particular types of cancer. LRRK2 mutations are rarely found in Alzheimer's disease (AD), but LRRK2 might play a part in tauopathies. The association of LRRK2 with the pathogenesis of apparently unrelated diseases remains enigmatic, but it might be related to the yet unknown diverse functions of LRRK2. Here, we reviewed current knowledge on the link between LRRK2 and several diseases, including PD, AD, CD, leprosy, and cancer, and discussed the possibility of targeting LRRK2 in such diseases. [BMB Reports 2015; 48(5): 243-248]
\end{abstract}

\section{GENERAL BIOLOGY OF LRRK2}

Implications from sequence analysis and homology modeling In 2004, two independent research groups identified leucine-rich repeat kinase 2 (LRRK2) or dardarin as a causative gene for autosomal dominant familial Parkinson's disease (PD) in multiple ethic families $(1,2)$. LRRK2 gene encodes a large protein of 2,527 amino acids with multiple functional domains, including protein-protein binding domains such as ankyrin-like repeats, leucine-rich repeats, and WD40 domains, and enzymatic domains such as Ras of complex (ROC) GTPase domain, a carboxyl-terminal of Roc (COR) domain, and a kinase domain. To date, physiological and pathological functions of LRRK2 are mainly linked to its GTPase and kinase activities.

${ }^{*}$ Corresponding author. Tel: +82-2-961-9381; Fax: +82-2-969-4570; E-mail: bdaelee@khu.ac.kr

http://dx.doi.org/10.5483/BMBRep.2015.48.5.032

Received 22 February 2015

Keywords: Leucine rich repeat kinase 2 (LRRK2), Parkinson's disease (PD), Alzheimer's disease (AD), Crohn's disease(CD)
On the basis of in silico sequence analysis and homology modeling, LRRK2 has been classified as a member of several protein subfamilies, such as ROC-COR (ROCO), mixed-lineage kinase (MLK), and receptor-interacting protein kinase (RIPK) families. On the basis of the well-defined cellular functions of such families, it is possible to predict plausible physiological and pathological functions of LRRK2. In mammals, the subfamily of ROCO, containing both ROC and COR domains, consists of four members, three of which are kinases, including LRRK2, its isoform LRRK1, and death-associated protein kinase (DAPK) (2). DAPK, a Ca ${ }^{2+} /$ calmodulin-regulated serine/threonine kinase, is implicated in the regulation of cell death, autophagy (3), cell adhesion and motility $(4,5)$, and inflammatory responses $(6,7)$. Loss of DAPK function was found in many primary cancer cells and cell lines, and the tumor progression and metastasis were inversely correlated with its expression (8). In addition to the homology of the ROC-COR domains in LRRK2 and DAPK, both kinases have been associated with the pathology of brain and neurodegenerative diseases. As mentioned above, $L R R K 2$ is a well-known PD gene, and genomewide association studies showed that single-nucleotide polymorphisms in DAPK1 are associated with late-onset Alzheimer's disease (AD).

$L R R K 2$ encodes a kinase domain with high sequence homology to the MLK subfamily of mitogen-activated protein kinase (MAPK) kinase kinase (MAPKKK). MAPKKK activates extracellular signal-regulated kinase (ERK) $1 / 2$, c-Jun $\mathrm{N}$-terminal kinase (JNK), and p38 proteins (9); and MLK-mediated activation of JNK has been associated with PD pathology. In the postmortem brain of PD patients, phospho-JNK staining was detected in neurons adjacent to midbrain neurons with Lewy bodies (10). An inhibitor of MLK, CEP-1347 was under clinical trials for PD patients (11). CEP-1347 prevented JNK activation in neurons and showed neuroprotection in a variety of PD and neurodegenerative models, but the drug failed to show efficacy in clinical trials.

The kinase domain of LRRK2 is closely related to RIPKs, which have a role in the recognition of damage-, pathogen-, or stress-associated signaling. RIPK family consists of seven members, including LRRK1 and LRRK2 being designated as RIPK6 and RIPK7, respectively. RIPK1-5 proteins are related to immune signaling pathways $(12,13)$. Extracellular pathogens are 
recognized by leucine-rich repeat domains of nucleotide-binding oligomerization domain-containing proteins (NODs). NOD1 and NOD2 are components of the innate immune system and are involved in sensing the presence of pathogens. Upon pathogen recognition, NODs recruit RIPK, which triggers downstream signaling through the activation of NF- $\kappa \mathrm{B}$, resulting in the production of inflammatory cytokines. LRRK2 has also been implicated in inflammatory responses downstream of Toll-like receptor (TLR), which functions as an extracellular pathogen sensor, similar to NOD (14). Moreover, LRRK2 is known to regulate NF-kB activity and the production of cytokines and reactive oxygen species (ROS) in immune cells (15).

Implications from the expression and localization of LRRK2 LRRK2 is ubiquitously expressed in various tissues, and it is most abundant in the brain, the kidney, and the immune system. Expression of LRRK2 in various tissues implies that LRRK2 might have diverse roles. To understand physiological roles of LRRK2, several animal models have been developed. Studies from LRRK2 knock-out animals suggest that LRRK2 might play a part in synaptic transmission. In Drosophila models, LRRK2 mutants have impaired synaptic structures, such as synapse-overgrowth, expanded nerve terminal, and increased number of synaptic boutons (16). Such phenotypes are thought to result from dysfunction of protein synthesis, mitochondria transport, and microtubule polymerization. In LRRK2 knock-out mice, the number of mature spines is decreased and synaptic transmission is altered in striatal projected neurons (17).

In addition to brain dysfunction, abnormal phenotypes were found in the kidneys of LRRK2 knock-out mice (18-20). In the absence of LRRK2, aggregates of $\alpha$-synuclein and ubiquitinated proteins were detected in the kidney; and autophagy-lysosomal pathway was also impaired, as indicated by the abnormal accumulation of lipofuscin granules and altered levels of LC3-II and p62, which are the markers for autophagy $(18,19)$. These studies suggest that LRRK2 might be involved in the regulation of protein homeostasis. It is unclear why the autophagic defect was found specifically in the kidney, but the higher expression level of LRRK2 in the kidney, possible compensation by LRRK1, the different rate of basal autophagy in distinct tissues, or a combination of such factors might provide an explanation.

LRRK2 is also highly expressed in immune cells, such as B-lymphocytes, monocytes, dendritic cells, and macrophages $(14,15,21,22)$. In the peripheral blood mononuclear cell population, LRRK2 is highly expressed in mature or activated monocytes, and the inhibition of LRRK2 has been shown to block monocyte maturation (21). Moreover, expression of LRRK2 could be upregulated in response to IFN- $\gamma$ and LPS in monocytes and macrophages $(14,21,22)$. These results suggest that LRRK2 might be involved in monocyte maturation and innate immune responses.

\section{LRRK2 IN HUMAN DISEASES}

Pathogenic mutations in $L R R K 2$ are the most prevalent genetic cause of $\mathrm{PD}$, but growing evidence suggest that $L R R K 2$ is implicated in other seemingly unrelated human diseases, such as inflammatory diseases, leprosy, and several types of cancer. However, the pathological functions of LRRK2 in such diseases are unclear. The following sections describe current knowledge on the linkage between LRRK2 and multiple human diseases, based on possible cellular functions of LRRK2 including regulation of cytoskeletal reorganization, vesicle trafficking, protein synthesis, protein homeostasis, autophagy, and inflammation.

\section{Parkinson's disease}

$\mathrm{PD}$ is the most popular neurodegenerative movement disorder characterized by resting tremor, bradykinesia, posture instability, and rigidity. General pathological features of PD include progressive loss of dopaminergic neurons in the substantia nigra and the presence of Lewy Bodies (LBs), intraneuronal abnormal protein deposits. Although etiology of PD is not clearly understood, multiple genetic risk loci have been identified. Mutations in $L R R K 2$ are the most popular genetic cause of PD, accounting for $4 \%$ of familial PD and $1 \%$ of sporadic PD across all populations. Carriers with pathogenic mutations in $L R R K 2$ possess typical, idiopathic PD with few clinical differences from non-carrier patients, and they develop various neuropathological features ranging from pure nigral degeneration without LBs to nigral degeneration with LBs (in brain stem or widespread), ubiquitin-positive inclusions or neurofibrillary tau-positive tangles $(2,23)$. Mutations in a single gene can bring about multiple pathological consequences.

The pathological functions of LRRK2 have mainly been associated with aberrant kinase activity. In general, high kinase activity of LRRK2 pathogenic mutants have been linked to pathological features of PD, such as dopaminergic neuronal cell death, impaired dopamine neurotransmission and locomotive activity, defects in protein synthesis and degradation, inflammatory responses, and oxidative damage (24-27). Therefore, identifying physiological substrates of LRRK2 kinase has been the subject of extensive investigation to provide explanation for the disturbed cellular functions of LRRK2 as well as to develop possible therapeutic interventions for the disease.

Synaptic dysfunction and aberrant kinase activity of LRRK2 have been reported in several animal models of PD. Recently, a study has suggested a role for LRRK2 at the synapse and provided evidence that endothilin A (EndoA) is a direct target of LRRK2 kinase activity (28). EndoA is a protein harboring a Bin/Amphiphysin/Rvs (BAR) domain that interacts with membranes and induces membrane deformation. In a Drosophila model, LRRK2 has been shown to affect synaptic transmission by phosphorylating EndoA, and consequently, control membrane deformation and endocytosis of synaptic membrane at Drosophila neuromuscular junctions. 
LRRK2 has also been shown to phosphorylate eukaryotic initiation factor $4 \mathrm{E}$ (elF4E)-binding protein (4E-BP) and ribosomal protein S15 (RPS15). Evidence for LRRK2-mediated phosphorylation of $4 \mathrm{E}-\mathrm{BP}$ and RPS15 raised the possibility that LRRK2 might play a part in the regulation of protein synthesis $(24,29)$. LRRK2-induced phosphorylation of 4E-BP dissociates 4E-BP from elF4E, and thereby increases free elF4E available for initiation of translation. It is plausible to suggest that increased and perhaps uncontrolled translation increases toxicity. Phosphorylation of RPS15 stimulates both cap-dependent and -independent mRNA translation, and thereby induces a bulk increase in protein synthesis, which again might induce neural toxicity.

In addition to protein synthesis, LRRK2 has also been implicated in the autophagy-lysosomal pathway. In animal and cell models of pathogenic LRRK2 mutants and in fibroblasts derived from PD patients with G2019S mutation, ubiquitinated proteins were aberrantly increased and enlarged aggregates of lysosomes were detected $(30,31)$. LRRK2 has also been shown to affect Rab7-dependent perinuclear lysosome clustering and lysosomal degradation (32). In addition, evidence for deregulation of autophagy has been provided by examining the brains of PD patients. Markers for autophagy were altered and autophagic vacuoles were accumulated in LRRK2 patient-derived cells (33). These lines of evidence suggest that impaired protein synthesis and degradation of the autophagy-lysosomal pathway might be responsible for LRRK2 pathology.

\section{Alzheimer's disease}

$A D$ is the most common neurodegenerative disease showing progressive and irreversible loss of memory and cognitive functions, ultimately leading to dementia and death. General pathological features of $A D$ include the presence of amyloid $\beta$ plaques and intraneuronal tangles of hyperphosphorylated forms of microtubule-associated protein Tau. In general, clinical symptoms of PD and AD are quite distinct, but PD and AD share some common pathological features, such as abnormal protein deposits. More than 50 percent of $A D$ patients have $\alpha$-synuclein-deposited LBs and tau deposits as in PD patients (34). Especially, $\alpha$-synuclein is involved in the pathogenesis of AD. The level of $\alpha$-synuclein was increased in the brain of $\mathrm{AD}$ patients as compared with age-matched control brains (35). In the early stages of AD, $\alpha$-synuclein is accumulated in presynaptic termini, implicating that aberrant deposits might be involved in the early events of AD pathogenesis (36). Furthermore, LBs and Lewy neurites are detected in the brains of $A D$ patients (37). The expression analysis revealed that $\alpha$-synuclein and LRRK2 mRNA levels were significantly increased in the temporal cortex of LBs variants of AD (LBV/AD) brains as compared with AD controls (38). Furthermore, $\alpha$-synuclein aggregation was increased in aged, LRRK2-null mice as compared with age-matched control $(18,39)$; and $\alpha$-synuclein-mediated cytotoxicity was exacerbated in $\alpha$-synuclein A53T and LRRK2 G2019S double transgenic mice as compared to $\alpha$-synuclein A53T mice (39). These results sug- gest that LRRK2 regulates $\alpha$-synuclein-mediated Lewy pathology in AD. Previous studies suggest that LRRK2 might be involved in tauopathies, which include AD. Pathogenic mutants of LRRK2, G2019S, and I2020T have been shown to directly phosphorylate Tau at Thr181, depending on the association between LRRK2 and $\beta$-tubulin (40); and in LRRK2 R1441G transgenic mouse brains, Tau phosphorylation was elevated (41). Increased Tau phosphorylation has also been detected in postmortem brains of PD patients with LRRK2 mutation (2). Hyperphosphorylated Tau is a general pathological feature of AD patients. Phosphorylated forms of Tau dissociate from tubulin and preferentially self-aggregate to form intraneural tangle, which is associated with neuronal toxicity and $A D$ pathology. Despite the molecular and cellular connections between proteins involved in AD pathology and LRRK2, LRRK2 mutations are not common among $A D$ patients. To date, pathogenic mutations of LRRK2 found in PD have not been found in $A D$ of various ethnic groups. A genome-wide association (GWA) study failed to identify common genetic risk factors for PD and AD (42). Thus, LRRK2 might not be a direct genetic risk factor for $A D$, but LRRK2 appears to be connected with several key proteins involved in AD pathology. LRRK2 might regulate protein homeostasis through regulation of lysosomal and autophagy pathways in neurodegenerative diseases, such as PD and AD.

\section{Inflammatory bowel disease}

GWA studies for Crohn's disease (CD) and leprosy suggest possible association between LRRK2 and inflammatory diseases $(43,44)$. (Leprosy will be discussed in the following section.) $C D$ is a chronic inflammatory bowel disease caused by dysfunction of immune response to commensal intestinal microbiota (45). Several single nucleotide polymorphisms (SNPS) in LRRK2 loci were identified as risk factors for CD (43). Moreover, LRRK2 mRNA level was highly increased in the inflamed biopsy sample, as compared to non-inflamed control from the same patient with CD; and LRRK2 protein was expressed in lamina propria macrophages, dendritic cells, and B lymphocytes (15). In general, LRRK2 is highly expressed in B lymphocytes, monocytes, and dendritic cells. Especially, LRRK2 has been implicated in monocyte maturation and in the production of inflammatory cytokines during the course of innate immunity. A study analyzing the pattern of LRRK2 expression in monocyte subpopulations has shown that LRRK2 expression is higher in mature $\mathrm{CD} 14^{+} \mathrm{CD} 16^{+}$cells than in $\mathrm{CD} 14^{+} \mathrm{CD} 16^{-}$monocytes (21). In addition, monocyte-maturing stimuli, such as IFN- $\gamma$, serum free condition, and cold stress induced LRRK2 expression, and pharmacological inhibition of LRRK2, by treating with small chemical inhibitors, attenuated monocyte maturation induced by IFN- $\gamma(21)$. Of note, IFN- $\gamma$ is a key cytokine in immune response and its concentration in plasma and intestinal mucosal are increased in $\operatorname{CD}(46,47)$.

A previous study using LRRK2-deficient mice has provided insights into the pathological mechanism of LRRK2 in in- 
flammatory bowel disease (48). LRRK2 negatively regulates NFAT1 activity by modulating its nuclear translocation. NFAT family comprises transcription factors that regulate innate immune response in T lymphocytes, macrophages, dendritic cells, and neutrophils. With the accompanying defects in NFAT signaling, LRRK2 deficient mice show hyperactive immune responses and enhanced susceptibility to dextran sulfate sodiuminduced inflammatory bowel disease. Notably, kinase activity of LRRK2 had no effect on NFAT1 activation. In particular, the inflammatory bowel disease risk allele M2397 affected the protein level of LRRK2, but had no effect on the kinase activity. Given that LRRK2 dysfunction in PD is mainly associated with the aberrant kinase activity, LRRK2 pathology in PD and inflammatory bowel disease might involve distinct molecular players and cellular pathways.

\section{Leprosy}

Leprosy is a chronic infectious disease caused by Mycobacterium leprae (M.leprae). Host immune responses and several genetic factors have been suggested to influence the clinical spectrum of and susceptibility to leprosy. Through GWA studies, significant associations between leprosy and SNPs in the genes encoding histocompatibility complex class II DR, RIPK2, tumor necrosis factor superfamily 15, C13orf31, and NOD2 have been found, and a trend toward an association with SNPs in LRRK2 has been reported (44). A previous study has provided evidence that NOD2-mediated innate immune signaling is associated with the pathology of leprosy. NOD2 functions as a pattern recognition receptor that senses bacterial-cell-wall peptidoglycan and muramyl dipeptide motif (49). Upon ligand binding, NOD2 receptor recruits RIPK2, a kinase which has high homology with LRRK2 in the kinase domain. RIPK2 recruitment initiates intracellular signaling pathways, leading to the activation of NF- $\mathrm{KB}$ signaling and induction of downstream target genes. Mice lacking NOD2 or RIPK2 are highly susceptible to bacterial infection, owing to defects in the production of inflammatory cytokines that activate T lymphocytes. Exactly how LRRK2 is linked to the pathology of leprosy remains unknown, but TLR signaling pathway is likely to be involved. Similar to NOD2 signaling pathways, TLR-mediated signaling is also linked to leprosy, and TLR1, 2, and 4 have genetic associations with leprosy. TLRs mediate pathogen sensing during innate immune responses and TLR1, 2, and 6 are involved in M. Leprae antigen recognition (50). Notably, TLR2, 4, 5, 6, 7, and 9 agonists, which signal via MYD88 pathway, enhance phosphorylation of LRRK2 at Ser910 and Ser935, which is thought to represent the activation status of LRRK2 kinase activity in macrophages. However, NOD1 and NOD2 agonists failed to induce the phosphorylation of LRRK2 (14). Therefore, it is possible that LRRK2 is involved in the pathology of leprosy through TLR-mediated inflammatory signaling pathways, but not through NOD signaling. Together, these results suggest that specific inflammatory stimuli and pathological conditions regulate LRRK2 activity and functions in multiple immune diseases.

\section{Cancer}

Debate about the association between PD and cancer has a long history. Epidemiological studies suggest that PD patients are less susceptible to develop most types of cancers, but with some exceptions. However, since genetic factors of PD have been identified, the premise of cancer susceptibility in PD patients is changing. PD genes, such as $\alpha$-synuclein, Parkin, PINK1, DJ-1, and LRRK2 have been associated with particular types of cancer (51). Parkin was the first to be identified as a tumor suppressor gene among PD risk genes, and loss-of-function mutations in Parkin were found in several types of cancers. DJ-1 has been shown to down-regulate the expression of a tumor suppressor gene, PTEN, and a high level of DJ-1 was detected in various types of cancer. In several epidemiological studies, the most prominent pathogenic mutant of LRRK2, G2019S carriers showed increased incidence in nonskin cancers, hormone-related cancers, and breast cancers (52-54). In papillary renal and thyroid carcinomas, chromosomal amplification of LRRK2 was detected, and LRRK2 expression and receptor tyrosine kinase MET activity were highly correlated (55). Furthermore, depletion of LRRK2 disrupted the activation of MET and prevented tumor cell proliferation (55). Precise role of LRRK2 in cancer progression is yet to be defined, but a possible link might be found in the regulation of protein translation. LRRK2 interacts with elF4E and directly phosphorylates $4 \mathrm{E}-\mathrm{BP}$. This elF4E-4E-BP mediated protein translation controls a subset of mRNAs, including transcription factors $E 2 F$ and $D P$, and phospho-4E-BP enhances their translation $(56,57)$. In mitotic and post-mitotic cells, E2F and DP heterodimerize and control cell fate in a distinct fashion. In mitotic cells, up-regulation of E2F-DP contributes to cancer progression by promoting cell proliferation, whereas in post-mitotic neurons, E2F-DP induces death. Notably, LRRK2 has been shown to negatively regulate the translational repression of E2F and DP through antagonizing miRNAs, let-7 and miR-184, respectively (56). Therefore, LRRK2 might have a role in cancer progression through the regulation of elF4E-4E-BP axis and miRNA-mediated protein translation. In addition to protein translation, regulation of autophagy and cytoskeletal rearrangement could play a part in LRRK2-mediated pathology in cancer.

\section{CONCLUSION}

Currently, it remains unclear how LRRK2 regulates the pathology of multiple, seemingly unrelated human diseases. LRRK2 is genetically associated with several diseases, implying that different mutations are associated with distinct pathology. Mutations can alter enzymatic activities of LRRK2 or the ability of LRRK2 to associate with its upstream regulators or downstream effectors. Indeed, pathogenic mutations linked to PD and cancer alter the kinase or GTPase activity of LRRK2; and accumulating lines of evidence suggest that enzymatic activities are critical to disease progression. However, mutations associated with immune dysfunction have little effect on the kin- 
ase activity of LRRK2, and the pathology is not linked to its kinase activity. Therefore, each disease associated with LRRK2 might involve distinct pathological mechanisms. To evaluate LRRK2 as a therapeutic target in multiple diseases, it will be of importance to understand the functions and signaling of LRRK2 in multiple diseases.

\section{ACKNOWLEDGEMENTS}

BDL is supported by the grants of NRF (NRF-2012R1A1A1010564 and NRF-2014R1A1A2055787) funded by Korea Ministry of Science.

\section{REFERENCES}

1. Paisan-Ruiz C, Jain S, Evans EW et al (2004) Cloning of the gene containing mutations that cause PARK8-linked Parkinson's disease. Neuron 44, 595-600

2. Zimprich A, Biskup S, Leitner P et al (2004) Mutations in LRRK2 cause autosomal-dominant parkinsonism with pleomorphic pathology. Neuron 44, 601-607

3. Bialik S and Kimchi A (2010) Lethal weapons: DAP-kinase, autophagy and cell death: DAP-kinase regulates autophagy. Curr Opin Cell Biol 22, 199-205

4. Kuo JC, Wang WJ, Yao CC, Wu PR and Chen RH (2006) The tumor suppressor DAPK inhibits cell motility by blocking the integrin-mediated polarity pathway. J Cell Biol 172, 619-631

5. Kuo JC, Lin JR, Staddon JM, Hosoya $\mathrm{H}$ and Chen RH (2003) Uncoordinated regulation of stress fibers and focal adhesions by DAP kinase. J Cell Sci 116, 4777-4790

6. Chuang YT, Fang LW, Lin-Feng MH, Chen RH and Lai MZ (2008) The tumor suppressor death-associated protein kinase targets to TCR-stimulated NF-kappa B activation. J Immunol 180, 3238-3249

7. Chuang YT, Lin YC, Lin KH et al (2011) Tumor suppressor death-associated protein kinase is required for full IL-1beta production. Blood 117, 960-970

8. Bialik S and Kimchi A (2006) The death-associated protein kinases: structure, function, and beyond. Annu Rev Biochem 75, $189-210$

9. Wang LH, Besirli CG and Johnson EM Jr (2004) Mixedlineage kinases: a target for the prevention of neurodegeneration. Annu Rev Pharmacol Toxicol 44, 451-474

10. Ferrer I, Blanco R, Carmona $M$ et al (2001) Active, phosphorylation-dependent mitogen-activated protein kinase (MAPK/ERK), stress-activated protein kinase/c-Jun N-terminal kinase (SAPK/JNK), and p38 kinase expression in Parkinson's disease and Dementia with Lewy bodies. J Neural Transm 108, 1383-1396

11. Parkinson Study G (2004) The safety and tolerability of a mixed lineage kinase inhibitor (CEP-1347) in PD. Neurology 62, 330-332

12. Zhang D, Lin J and Han J (2010) Receptor-interacting protein (RIP) kinase family. Cell Mol Immunol 7, 243-249

13. Meylan E and Tschopp J (2005) The RIP kinases: crucial integrators of cellular stress. Trends Biochem Sci 30, 151-159

14. Dzamko N, Inesta-Vaquera F, Zhang J et al (2012) The
IkappaB kinase family phosphorylates the Parkinson's disease kinase LRRK2 at Ser935 and Ser910 during Toll-like receptor signaling. PLoS One 7, e39132

15. Gardet A, Benita Y, Li C et al (2010) LRRK2 is involved in the IFN-gamma response and host response to pathogens. J Immunol 185, 5577-5585

16. Lee S, Liu HP, Lin WY, Guo H and Lu B (2010) LRRK2 kinase regulates synaptic morphology through distinct substrates at the presynaptic and postsynaptic compartments of the Drosophila neuromuscular junction. J Neurosci 30, 16959-16969

17. Parisiadou L, Yu J, Sgobio C et al (2014) LRRK2 regulates synaptogenesis and dopamine receptor activation through modulation of PKA activity. Nat Neurosci 17, 367-376

18. Tong $\mathrm{Y}$, Yamaguchi H, Giaime E et al (2010) Loss of leucine-rich repeat kinase 2 causes impairment of protein degradation pathways, accumulation of alpha-synuclein, and apoptotic cell death in aged mice. Proc Natl Acad Sci U S A 107, 9879-9884

19. Tong $\mathrm{Y}$, Giaime $\mathrm{E}$, Yamaguchi $\mathrm{H}$ et al (2012) Loss of leucine-rich repeat kinase 2 causes age-dependent bi-phasic alterations of the autophagy pathway. Mol Neurodegener 7,2

20. Herzig MC, Kolly C, Persohn E et al (2011) LRRK2 protein levels are determined by kinase function and are crucial for kidney and lung homeostasis in mice. Hum Mol Genet 20, 4209-4223

21. Thevenet J, Pescini Gobert R, Hooft van Huijsduijnen R, Wiessner C and Sagot YJ (2011) Regulation of LRRK2 expression points to a functional role in human monocyte maturation. PLoS One 6, e21519

22. Hakimi M, Selvanantham T, Swinton E et al (2011) Parkinson's disease-linked LRRK2 is expressed in circulating and tissue immune cells and upregulated following recognition of microbial structures. J Neural Transm 118, 795-808

23. Funayama M, Hasegawa K, Ohta E et al (2005) An LRRK2 mutation as a cause for the parkinsonism in the original PARK8 family. Ann Neurol 57, 918-921

24. Liu Z, Wang X, Yu Y et al (2008) A Drosophila model for LRRK2-linked parkinsonism. Proc Natl Acad Sci U S A 105, 2693-2698

25. Tong Y, Pisani A, Martella G et al (2009) R1441C mutation in LRRK2 impairs dopaminergic neurotransmission in mice. Proc Natl Acad Sci U S A 106, 14622-14627

26. Alegre-Abarrategui J, Christian H, Lufino MM et al (2009) LRRK2 regulates autophagic activity and localizes to specific membrane microdomains in a novel human genomic reporter cellular model. Hum Mol Genet 18, 4022-4034

27. Chen CY, Weng YH, Chien KY et al (2012) (G2019S) LRRK2 activates MKK4-JNK pathway and causes degeneration of $\mathrm{SN}$ dopaminergic neurons in a transgenic mouse model of PD. Cell Death Differ 19, 1623-1633

28. Matta S, Van Kolen K, da Cunha R et al (2012) LRRK2 controls an EndoA phosphorylation cycle in synaptic endocytosis. Neuron 75, 1008-1021

29. Martin I, Kim JW, Lee BD et al (2014) Ribosomal protein s15 phosphorylation mediates LRRK2 neurodegeneration in Parkinson's disease. Cell 157, 472-485

30. MacLeod D, Dowman J, Hammond R, Leete T, Inoue K 
and Abeliovich A (2006) The familial Parkinsonism gene LRRK2 regulates neurite process morphology. Neuron 52, 587-593

31. Hockey LN, Kilpatrick BS, Eden ER et al (2015) Dysregulation of lysosomal morphology by pathogenic LRRK2 is corrected by TPC2 inhibition. J Cell Sci 128, 232-238

32. Dodson MW, Zhang T, Jiang C, Chen S and Guo M (2012) Roles of the Drosophila LRRK2 homolog in Rab7dependent lysosomal positioning. Hum Mol Genet 21, 1350-1363

33. Sanchez-Danes A, Richaud-Patin Y, Carballo-Carbajal I et al (2012) Disease-specific phenotypes in dopamine neurons from human iPS-based models of genetic and sporadic Parkinson's disease. EMBO Mol Med 4, 380-395

34. Guo JL, Covell DJ, Daniels JP et al (2013) Distinct alpha-synuclein strains differentially promote tau inclusions in neurons. Cell 154, 103-117

35. Kim S, Seo JH and Suh YH (2004) Alpha-synuclein, Parkinson's disease, and Alzheimer's disease. Parkinsonism Relat Disord 10 Suppl 1, S9-13

36. Culvenor JG, McLean CA, Cutt S et al (1999) Non-Abeta component of Alzheimer's disease amyloid (NAC) revisited. NAC and alpha-synuclein are not associated with Abeta amyloid. Am J Pathol 155, 1173-1181

37. Koehler NK, Stransky E, Shing M et al (2013) Altered serum IgG levels to alpha-synuclein in dementia with Lewy bodies and Alzheimer's disease. PLoS One 8, e64649

38. Linnertz C, Lutz MW, Ervin JF et al (2014) The genetic contributions of SNCA and LRRK2 genes to Lewy Body pathology in Alzheimer's disease. Hum Mol Genet 23, 4814-4821

39. Lin X, Parisiadou L, Gu XL et al (2009) Leucine-rich repeat kinase 2 regulates the progression of neuropathology induced by Parkinson's-disease-related mutant alpha-synuclein. Neuron 64, 807-827

40. Kawakami F, Yabata T, Ohta E et al (2012) LRRK2 phosphorylates tubulin-associated tau but not the free molecule: LRRK2-mediated regulation of the tau-tubulin association and neurite outgrowth. PLoS One 7, e30834

41. Li Y, Liu W, Oo TF et al (2009) Mutant LRRK2(R1441G) BAC transgenic mice recapitulate cardinal features of Parkinson's disease. Nat Neurosci 12, 826-828

42. Moskvina V, Harold D, Russo G et al (2013) Analysis of genome-wide association studies of Alzheimer disease and of Parkinson disease to determine if these 2 diseases share a common genetic risk. JAMA Neurol 70, 1268-1276

43. Barrett JC, Hansoul S, Nicolae DL et al (2008) Genomewide association defines more than 30 distinct susceptibility loci for Crohn's disease. Nat Genet 40, 955-962

44. Zhang FR, Huang W, Chen SM et al (2009) Genomewide association study of leprosy. N Engl J Med 361, 2609-2618
45. Xavier RJ and Podolsky DK (2007) Unravelling the pathogenesis of inflammatory bowel disease. Nature 448, 427-434

46. Fuss IJ, Neurath $M$, Boirivant $M$ et al (1996) Disparate CD4 + lamina propria (LP) lymphokine secretion profiles in inflammatory bowel disease. Crohn's disease LP cells manifest increased secretion of IFN-gamma, whereas ulcerative colitis LP cells manifest increased secretion of IL-5. J Immunol 157, 1261-1270

47. Niessner M and Volk BA (1995) Altered Th1/Th2 cytokine profiles in the intestinal mucosa of patients with inflammatory bowel disease as assessed by quantitative reversed transcribed polymerase chain reaction (RT-PCR). Clin Exp Immunol 101, 428-435

48. Liu Z, Lee J, Krummey S, Lu W, Cai $\mathrm{H}$ and Lenardo MJ (2011) The kinase LRRK2 is a regulator of the transcription factor NFAT that modulates the severity of inflammatory bowel disease. Nat Immunol 12, 1063-1070

49. Franchi L, Warner N, Viani K and Nunez G (2009) Function of Nod-like receptors in microbial recognition and host defense. Immunol Rev 227, 106-128

50. Krutzik SR, Ochoa MT, Sieling PA et al (2003) Activation and regulation of Toll-like receptors 2 and 1 in human leprosy. Nat Med 9, 525-532

51. Devine MJ, Plun-Favreau H and Wood NW (2011) Parkinson's disease and cancer: two wars, one front. Nat Rev Cancer 11, 812-823

52. Agalliu I, San Luciano M, Mirelman A et al (2015) Higher Frequency of Certain Cancers in LRRK2 G2019S Mutation Carriers With Parkinson Disease: A Pooled Analysis. JAMA Neurol 72, 58-65

53. Inzelberg R, Cohen OS, Aharon-Peretz J et al (2012) The LRRK2 G2019S mutation is associated with Parkinson disease and concomitant non-skin cancers. Neurology 78, 781-786

54. Saunders-Pullman R, Barrett MJ, Stanley KM et al (2010) LRRK2 G2019S mutations are associated with an increased cancer risk in Parkinson disease. Mov Disord 25, 2536-2541

55. Looyenga BD, Furge KA, Dykema KJ et al (2011) Chromosomal amplification of leucine-rich repeat kinase-2 (LRRK2) is required for oncogenic MET signaling in papillary renal and thyroid carcinomas. Proc Natl Acad Sci U S A 108, 1439-1444

56. Gehrke S, Imai Y, Sokol N and Lu B (2010) Pathogenic LRRK2 negatively regulates microRNA-mediated translational repression. Nature 466, 637-641

57. Gingras AC, Raught B and Sonenberg N (1999) elF4 initiation factors: effectors of mRNA recruitment to ribosomes and regulators of translation. Annu Rev Biochem 68, 913-963 